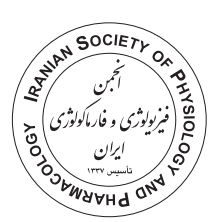

\title{
Antimicrobial potential of a gel containing hydrogen peroxide and hyaluronic acid
}

\author{
Jyothi Tadakamadla $^{1^{*}}(\mathbb{D})$, Elisa Boccalari ${ }^{2}$, Cinzia Maspero $^{2}$
}

1. School of Medicine and Dentistry, Griffith University, Gold Coast, QLD, Australia

2. Department of Biomedical, Surgical and Dental Sciences, School of Dentistry, University of Milan; Milan, Italy

\section{Dear Editor,}

Although biocidal action of hydrogen peroxide (HPO) is not well understood, many authors believe that the free hydroxyl radicals causes oxidation of DNA, proteins and lipid membranes (Linley et al., 2012). Use of HPO on skin problems is recently gaining importance due to its efficacy in preventing acne (Milani et al., 2003; Veraldi et al., 2016). It is also being used in treating Molluscum contagiosum due to its antiviral properties (Schianchi et al., 2018). Hyaluronic acid (HA) is another product with promising skin benefits because of its moisturising and wound healing properties, along with its ability to prevent wrinkles (Lee et al., 2015). It also produces extracellular matrix and prevents infections by modulating cellular immunity (Jegasothy et al., 2014). There are also clinical studies that tested the effectiveness of HA on skin, particularly in decreasing the depth of wrinkles, hydrating the skin and improving the firmness and elasticity of the skin (Jegasothy et al., 2014; Lee et al., 2015; Pavicic et al., 2011).

The sensitivity of hyaluronan polysaccharidic chain to radical degradation promoted by HPO decomposition, alone or in presence of metal ions like iron and copper is well known. Nevertheless, combination of these two molecules have been used to develop therapeutical approaches against tumour growth, thanks to the viscosity of HA in water solution, which facilitates slower rate of degradation of HPO in vivo (Abbasi et al., 2021; Aki- ma et al., 2016). As a consequence, oxygen delivery occurs for longer time and locally, improving efficacy and allowing to use lower dosage of HPO, less than the common 3\% used in antiseptic application. Such lower concentrations of HPO undoubtedly have advantages in terms of cell viability of the tissues treated and reduction in polysaccharide degradation. Therefore, a formulation combining both these products was developed and tested on common skin bacteria and Candida albicans.

To our knowledge, we could not find any research that tested the effectiveness of this combination through in vitro or in vivo studies. However, a combination of HPO with other agents, such as retinoids, has been tested. A Randomised Clinical Trial testing a combination of HPO and adapalene found it to have a comparable effectiveness on skin acne and more tolerability than benzoyl peroxide combined with adapalene (Capizzi et al., 2004). We tested the effectiveness of the novel combination of HPO and HA as gel (Ialuxid, BMG Pharma company, Italy; composition: aqua, hydrogen peroxide, carbomer, xanthan gum, hydroxyethyl acrylate / sodium acryloydimethyl taurate copolymer, squalane, glycine, sodium hyaluronate, polysorbate 60 , oxyquinoline sulfate) in comparison to neomycin and a control group (glycerol) on several microbial strains including Cutibacterium acnes, Staphylococcus aureus, Staphylococcus epidermidis, Streptococcus pyogenes, Streptococcus Gordonii, Pseudomonas aeruginosa, Klebsiella pneumoniae and

* Corresponding author: Jyothi Tadakamadla, Jyothi.tadakamadla@griffithuni.edu.au

Received 22 July 2020; Revised from 7 October 2020; Accepted 27 October 2020

Citation: Tadakamadla J, Elisa Boccalari E, Maspero C. Antimicrobial potential of a gel containing hydrogen peroxide and hyaluronic acid. Physiology and Pharmacology 2021; 25: 189-193. http://dx.doi.org/10.52547/ppj.25.3.189 
TABLE 1: Growth conditions and Inoculum concentration of each microbial strain used in this analysis.

STRAIN

Growth conditions
Inoculum concentration

(CFU/plate)

Pseudomonas aeruginosa

ATCC 9027

Casein Soya Bean Digest Agar $\left(3-4\right.$ days $\left.-37^{\circ} \mathrm{C}\right)$

$2.5 \times 10^{3}$

Staphylococcus aureus

ATCC 6538

Casein Soya Bean Digest Agar $\left(3-4\right.$ days $\left.-37^{\circ} \mathrm{C}\right)$

$6.0 \times 10^{3}$

Staphylococcus epidermidis

ATCC 12228

Casein Soya Bean Digest Agar $\left(3-4\right.$ days $\left.-37^{\circ} \mathrm{C}\right)$

$3.5 \times 10^{3}$

Streptococcus pyogenes

ATCC 12344

Casein Soya Bean Digest Agar

(5-6 days $-37^{\circ} \mathrm{C}-$ Anaerobiosis)

$4.5 \times 10^{3}$

Streptococcus gordonii

ATCC 10558

Casein Soya Bean Digest Agar

(3-4 days $-37^{\circ} \mathrm{C}-$ microaerohilic)

$3.7 \times 10^{4}$

Klebsiella pneumoniae ATCC 4352

Casein Soya Bean Digest Agar (3-4 days $\left.-37^{\circ} \mathrm{C}\right)$

$7.4 \times 10^{3}$

Propionibacterium acnes

ATCC 6919

Casein Soya Bean Digest Agar (3-4 days $\left.-37^{\circ} \mathrm{C}\right)$

$6.8 \times 10^{4}$

Candida albicans

ATCC 10231

Sabouraud-dextrose agar $\left(3-4\right.$ days $\left.-25^{\circ} \mathrm{C}\right)$

$4.5 \times 10^{2}$

TABLE 2: Antimicrobial effectiveness of the diluted and undiluted test and control products on the common skin microbes.

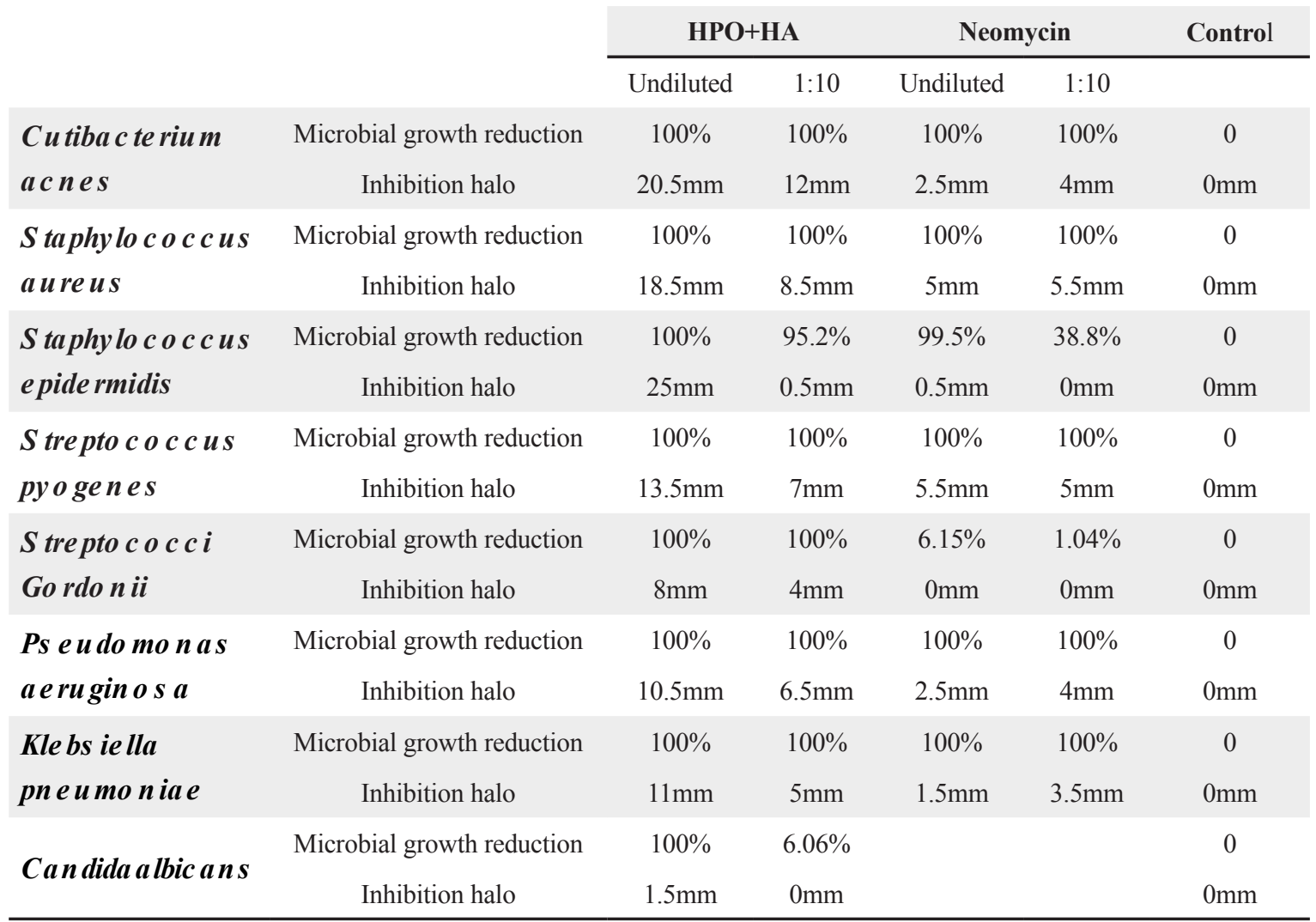


Candida albicans. As this was an in vitro experiment, no ethics approval was required. The $C$. acnes is the most common bacteria associated with surgical site contamination and resides in the deep dermal layer of the skin within pilosebaceous glands and hair follicle. Surgeons rely on antibiotics in an attempt to eradicate these bacteria preoperatively - antibiotics have been the treatment of choice, while HPO has recently been found to show promising results in preventing these bacteria (Hernandez et al., 2019). Although S. aureus and S. epidermidis are common pathogens on the skin, they are involved in opportunistic infections, particularly in patients with burns (Gallagher et al., 2007). The S. Pyogenes is also a common group A streptococcus that colonises nasopharynx, skin and oral cavity while $S$. gordonii is mostly found in the oral cavity (Nobbs et al., 2007). Candida species are common skin pathogens and C. albicans is most often associated with symptomatic skin infections (Kühbacher et al., 2017), interactions between candida and streptococci are also not uncommon (Koo et al., 2018). In addition, P. aeruginosa, which causes varying levels of skin and soft tissue infections, (Bassetti et al., 2018) and K. pneumoniae, less commonly reported in skin infections, were also considered (Chang et al., 2008).

To investigate antimicrobial activity, an inhibition contact test was conducted on solid-agar cultures. For this purpose, $25 \mathrm{~mm}$ cellulose discs were used as support for contact of the sample with the microbial cultures. Antimicrobial activity of the tests and control products was evaluated through the observation of the microbial growth reduction below the application area and by measuring the inhibition halo around it. The original lyophilized strain was grown in a suitable liquid growth medium and plated on solid medium by isolating individual colonies which were then amplified and frozen at $-30^{\circ} \mathrm{C}$ in aliquots of known titre. Preparation of the strains for the test was carried out starting from an aliquot thawed, taken up in liquid medium, isolated and then allowed to grow by incubating for specific conditions. Each strain was inoculated into a layer of agar surface at a concentration indicated in Table 1; the concentration of viable cells was determined by the method of the plate count.

Undiluted and diluted $(1: 10)$ product was placed on $25 \mathrm{~mm}$ cellulose supports until completely adsorbed and the supports were then placed on each of the inoculat- ed medium plates. Medium plates not inoculated and untreated were used to check sterility of the culture medium. The experiment was repeated when the medium plates couldn't be evaluated. Effect of HPO+HA, neomycin and control was tested on all strains except $C$. albicans, which was not tested with neomycin.

HPO+HA had excellent antimicrobial effect against all the bacterial strains, both when tested undiluted and diluted. However, its effect on C. albicans was only observed when undiluted (Table 2). Neomycin was also found to have excellent antimicrobial effect against $P$. aeruginosa, $S$. aureus, $S$. pyogenes, $K$. pneumoniae, $C$. acnes, both when tested undiluted and diluted while its effectiveness on S. epidermidis was only observed when tested undiluted, with a minimal antimicrobial effect when tested diluted. Neomycin did not present any antimicrobial effect against $S$. gordonii. Results from our preliminary in vitro research indicates that the new combination has promising role in skin infections due to its effectiveness on several common skin microbes. We are intending to conduct clinical studies to further establish its effectiveness.

\section{Acknowledgement: None}

\section{References}

Abbasi A, Pakravan N, Hassan Z M. Hyaluronic acid optimises therapeutic effects of hydrogen peroxide-induced oxidative stress on breast cancer. J Cell Physiol 2021; 236: 1494-514. https://doi.org/10.1002/jcp.29957

Akima R, Ogawa Y, Morita-Tokuhiro S, Tsuzuki A, Yaogawa S. New enzyme-targeting radiosensitizer (KORTUC) containing hydrogen peroxide \& sodium hyaluronate for intra-tumoral injection using mice transplanted with SCC VII tumor. Int J Cancer Clin Res 2016; 3: 48. https://doi. org/10.23937/2378-3419/3/2/1048

Bassetti M, Vena A, Croxatto A, Righi E, Guery B. How to manage Pseudomonas aeruginosa infections. Drugs Context 2018; 7. https://doi.org/10.7573/dic.212527

Capizzi R, Landi F, Milani M, Amerio P. Skin tolerability and efficacy of combination therapy with hydrogen peroxide stabilized cream and adapalene gel in comparison with benzoyl peroxide cream and adapalene gel in common acne. A randomized, investigator-masked, controlled trial. $\mathrm{Br} \mathrm{J}$ Dermatol 2004; 151: 481-4. https://doi.org/10.1111/j.13652133.2004.06067.x

Chang CM, Lee HC, Lee NY, Lee IW, Wu CJ, Chen PL, et 
al. Community-acquired Klebsiella pneumoniae complicated skin and soft-tissue infections of extremities: emphasis on cirrhotic patients and gas formation. Infection 2008; 36 : 328-34. https://doi.org/10.1007/s15010-008-7272-3

Gallagher JJ, Williams-Bouyer N, Villarreal C, Heggers JP, Herndon DN. Treatment of infection in burns. In: Herndon DN, editor. Total Burn Care. 3rd ed. Philadelphia: Elsevier, 2007, p. 136-76. https://doi.org/10.1016/B978-1-41603274-8.50015-5

Hernandez P, Sager B, Fa A, Liang T, Lozano C, Khazzam M. Bactericidal efficacy of hydrogen peroxide on Cutibacterium acnes. Bone Joint Res 2019; 8: 3-10. https://doi. org/10.1302/2046-3758.81.BJR-2018-0145.R1

Jegasothy SM, Zabolotniaia V, Bielfeldt S. Efficacy of a new topical nano-hyaluronic acid in humans. J Clin Aesthet Dermatol 2014; 7: 27.

Koo H, Andes DR, Krysan DJ. Candida-streptococcal interactions in biofilm-associated oral diseases. Plos Pathog 2018; 14: e1007342. https://doi.org/10.1371/journal. ppat. 1007342

Kühbacher A, Burger-Kentischer A, Rupp S. Interaction of Candida species with the skin. Microorganisms 2017; 5: 32. https://doi.org/10.3390/microorganisms5020032

Lee DH, Oh IY, Koo KT, Suk JM, Jung SW, Park JO, et al. Improvement in skin wrinkles using a preparation containing human growth factors and hyaluronic acid serum. J Cosmet Laser Ther 2015; 17: 20-3. https://doi.org/10.3109/ 14764172.2014 .968577
Linley E, Denyer SP, McDonnell G, Simons C, Maillard JY. Use of hydrogen peroxide as a biocide: new consideration of its mechanisms of biocidal action. J Antimicrob Chemother 2012; 67: 1589-96. https://doi.org/10.1093/jac/dks129

Milani M, Bigardi A, Zavattarelli M. Efficacy and safety of stabilised hydrogen peroxide cream (Crystacide) in mild-to-moderate acne vulgaris: a randomised, controlled trial versus benzoyl peroxide gel. Curr Med Res Opin 2003; 19: 135-8. https://doi.org/10.1080/030079903125001523

Nobbs AH, Shearer BH, Drobni M, Jepson MA, Jenkinson HF. Adherence and internalization of Streptococcus gordonii by epithelial cells involves $\beta 1$ integrin recognition by SspA and SspB (antigen I/II family) polypeptides. Cell Microbiol 2007; 9: 65-83. https://doi.org/10.1111/j.14625822.2006.00768.x

Pavicic T, Gauglitz GG, Lersch P, Schwach-Abdellaoui K, Malle B, Korting HC, et al. Efficacy of cream-based novel formulations of hyaluronic acid of different molecular weights in anti-wrinkle treatment. J Drugs Dermatol 2011; 10: 990-1000.

Schianchi R, Nazzaro G, Veraldi S. Treatment of molluscum contagiosum with hydrogen peroxide. Clin Exp Dermatol 2018; 43: 66-7. https://doi.org/10.1111/ced.13250

Veraldi S, Micali G, Berardesca E, Dall'Oglio F, Sinagra JL, Guanziroli E. Results of a multicenter, randomized, controlled trial of a hydrogen peroxide-based kit versus a benzoyl peroxide-based kit in mild-to-moderate acne. J Clin Aesthet Dermatol 2016; 9: 50. 